\title{
Perinatal Immune Activation Produces Persistent Sleep Alterations and Epileptiform Activity in Male Mice
}

\author{
Galen Missig', Emery L Mokler', James O Robbins', Abigail J Alexander', Christopher J McDougle² and \\ William A Carlezon Jr*,I
}

'Basic Neuroscience Division, Department of Psychiatry, Harvard Medical School, McLean Hospital, Belmont, MA, USA; ' ${ }^{2}$ urie Center for Autism, Department of Psychiatry, Harvard Medical School, Massachusetts General Hospital, Lexington, MA, USA

\begin{abstract}
Increasing evidence suggests a role for inflammation in neuropsychiatric conditions, including autism spectrum disorder (ASD). Previous work in rodents has established that immune activation during critical developmental periods can cause phenotypes that reproduce core features of ASD, including decreased social interaction, aberrant communication, and increased repetitive behavior. In humans, ASD is frequently associated with comorbid medical conditions including sleep disorders, motor hyperactivity, and seizures. Here we use a 'twohit' immune-activation paradigm to determine whether perinatal immune activation can also produce these comorbid features in mice. In this paradigm, we treated timed-pregnant mice with polyinosinic:polycytidylic acid (Poly l:C), which simulates a viral infection, on gestational day 12.5 according to an established maternal immune activation regimen. A subset of the offspring also received a second 'hit' of lipopolysaccharide (LPS), which simulates a bacterial infection, on postnatal day 9. At 6 weeks of age, mice were implanted with wireless telemetry transmitters that enabled continuous measurements of electroencephalography (EEG), electromyography (EMG), locomotor activity, and subcutaneous temperature. Effects at 7 and 12 weeks of age were compared. Both prenatal Poly I:C and postnatal LPS produced changes in locomotor activity and temperature patterns, increases in slow-wave sleep, and shifts in EEG spectral power, several of which persisted at 12 weeks of age. Postnatal LPS also produced persistent increases in spontaneous bursts of epileptiform activity (spike-wave discharges) that occurred predominantly during sleep. Our findings demonstrate that early-life immune activation can lead to long-lasting physiologic perturbations that resemble medical comorbidities often seen in ASD and other neuropsychiatric conditions. Neuropsychopharmacology (2018) 43, 482-49I; doi:I0.1038/npp.2017.243; published online 15 November 2017
\end{abstract}

\section{INTRODUCTION}

Accumulating evidence suggests that the immune system is involved in psychiatric illness (Miller et al, 2017). As an example, there appear to be connections between immune system dysregulation and autism spectrum disorder (ASD), including higher rates of ASD in families with a history of autoimmune disorders (Wu et al, 2015). In addition, maternal infection or fever during pregnancy increases the risk that offspring will develop ASD (Hornig et al, 2017; Jiang et al, 2016), and individuals with ASD often have higher levels of systemic proinflammatory cytokines (Masi et al, 2015). A transcriptomic analysis of post-mortem brain samples from individuals with ASD revealed an upregulation of a cluster of immune-response genes (Gupta et al, 2014). This confluence of evidence has led to the hypothesis that an immune-based subtype of ASD could exist (McDougle et al, 2015) and account for some-but not all-cases of this condition. This possibility is important to evaluate because

* Correspondence: Dr WA Carlezon, Basic Neuroscience Division, Department of Psychiatry, Harvard Medical School, McLean Hospital, MRC 217, I I 5 Mill Street, Belmont, MA 02478, USA, Tel: + I 617855 2021, Fax: + 617855 2023, E-mail: bcarlezon@mclean.harvard.edu Received 7 August 2017; revised 17 September 2017; accepted 2 October 2017; accepted article preview online 6 October 2017 an immune-related subtype of ASD may be sensitive to treatment strategies that would not be effective, or even cause harm, in subtypes with other pathophysiologies.

Laboratory animal models of early-life immune activation suggest a causal role for immune dysregulation in ASD. It has been shown repeatedly that maternal immune activation (MIA) in rodents and primates results in offspring with behavioral alterations in the three core symptom domains of ASD, including decreased social interaction, aberrant communication, and increased repetitive or focused behavior (Machado et al, 2015; Malkova et al, 2012; Smith et al, 2007). MIA may act as a 'disease primer', by making an organism more susceptible to negative effects of environmental exposures or genetic mutations (Estes and McAllister, 2016). Consistent with this hypothesis, it has been reported that peripubertal stress can unmask or exacerbate the behavioral and neurochemical consequences of MIA (Yin et al, 2013). To further explore this possibility in mice, we have developed a 'two-hit' early-life immune activation model, in which offspring receive an initial immune challenge on embryonic day 12.5 (E12.5) with polyinosinic: polycytidylic acid (Poly I:C) - a toll-like receptor-3 (TLR3) agonist that simulates a viral infection-and then a second 'hit' with lipopolysaccharide (LPS) - a TLR4 agonist that simulates a bacterial infection-on postnatal day 9 (PND9) 
(Kawai and Akira, 2010). This paradigm enables studies of whether MIA increases the susceptibility of offspring to the effects of a second immune challenge early in development. Importantly, early developmental exposure to LPS within this timeframe is sufficient to cause a variety of behavioral changes in mice that resemble some of the diagnostic features of ASD (Custodio et al, 2017). We have reported preliminary findings (Missig et al, 2016) indicating that our combined ('two-hit') approach produces behavioral alterations that resemble the core features of ASD and are broadly consistent with previous reports (Custodio et al, 2017; Smith et al, 2007).

In humans, the behavioral signs of ASD are frequently associated with comorbid medical conditions, including sleep disorders, motor hyperactivity, and seizures (Jeste, 2011). Interestingly, an estimated $25-33 \%$ of people with ASD have comorbid seizure disorders (Canitano, 2007), with a higher prevalence of epileptiform activity even in the absence of a diagnosed seizure disorder (Chez et al, 2006). These comorbid conditions are reflected by alterations in highly objective end points that are similar-if not identical - to those that can be measured in laboratory animals, including mice (Wells et al, 2017). The use of end points that are homologous across species will facilitate the implementation of translational studies that identify pathophysiologies, better predict clinical outcomes in humans, improve the success of clinical trials, and facilitate the development of more effective therapeutics (Lezak et al, 2017).

The present study was designed to examine the effects of perinatal immune activation in mice on a set of highly objective, translationally relevant end points that reflect physiological disturbances that are frequently comorbid with the core diagnostic domains of ASD. We used a wireless transmitter system that provides uninterrupted telemetry including EEG (electroencephalography), EMG (electromyography), locomotor activity, and subcutaneous temperature in freely behaving (untethered) mice for up to 6 weeks. These data were used to quantify immune activation-related changes in sleep/wake patterns, epileptiform activity, and EEG spectral power, as well as activity and temperature. To examine the persistence of the effects, two time points were compared, separated by 5 weeks. Considering the higher prevalence of ASD in males (Werling and Geschwind, 2013), only male mice were examined in the present studies. Because sleep and circadian rhythms can be profoundly influenced by interactions with conspecifics, investigators, and/or behavioral testing procedures, the mice were singly housed and left undisturbed for the data collection portions of this study.

\section{MATERIALS AND METHODS}

\section{Mouse Model of Maternal and Postnatal Immune Activation}

Timed pregnancies in C57BL/6 J mice (6-8 weeks, Jackson Laboratories, Bar Harbor, ME) were implemented by housing a female and male pair overnight. The following morning, pairs were separated and mid-day was considered embryonic day 0.5 (E0.5). Pregnancy was identified by body weight gain and physical appearance. At E12.5 pregnant dams received intraperitoneal (IP) injections of either $20 \mathrm{mg} /$

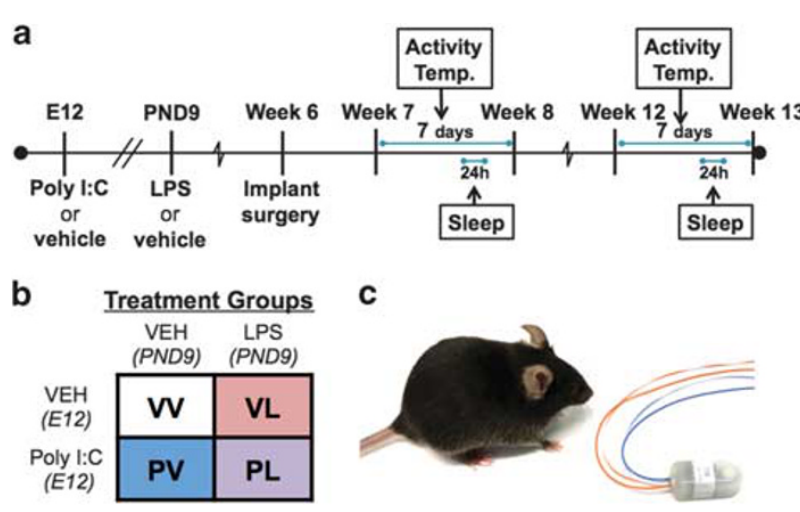

Figure I Experimental design of the studies. (a) Two time periods were examined: 7 and 12 weeks of age. (b) Pregnant dams received Poly l:C or vehicle on EI 2, and offspring LPS or vehicle on PND9; total N=6/group. (c) Male offspring were implanted subcutaneously with wireless transmitters to enable uninterrupted data collection.

kg Poly I:C ('P') (Catalog \#P9852, Sigma-Aldrich, St Louis, MO, USA) or vehicle ('V') (sterile phosphate-buffered saline). Dams were monitored daily for parturition. On PND9, pups received subcutaneous injections of either $10 \mathrm{mg} / \mathrm{kg}$ of LPS ('L') from Escherichia coli 0111:B4 (Catalog \#L3024, Sigma-Aldrich) or endotoxin-free saline ('V'), yielding four different treatment groups (VV, PV, VL, PL) (Figures 1a and b). Offspring were group-housed until surgery with mice of the same sex and treatment $(n=6 /$ group). Following transmitter implantation surgery (Figure 1c), mice were singly housed with both food and water provided ad libitum in an isolated vivarium room kept at $22{ }^{\circ} \mathrm{C}\left( \pm 2{ }^{\circ} \mathrm{C}\right)$ with a relative humidity of $50 \%( \pm 20 \%)$ on a 12-h light-dark cycle $(0700-1900 \mathrm{~h})$. All experiments were approved by McLean Hospital Institutional Animal Care and Use Committee following guidelines set by the National Institutes of Health.

\section{EEG/EMG Transmitter Implantation}

At 6 weeks of age and $\geqslant 20$ gm body weight, mice were anesthetized with ketamine/xylazine $(100 / 10 \mathrm{mg} / \mathrm{kg}$, IP). Each mouse was implanted with a wireless PhysioTel F20EET (Data Sciences International (DSI), St Paul, MN, USA) biotelemetry transmitter as described (Wells et al, 2017). In brief, mice were immobilized in a stereotaxic instrument and a small incision was made from the medial skull region to the posterior of the neck. The transmitter was placed in the subcutaneous pocket on the posterior of the mouse aligned with the spine between the forelimb and hind limb. Two biopotential leads (EMG electrodes) were then threaded through the cervical trapezius muscle via a small incision made by a $20-G$ needle and held in place with nondissolvable silk sutures. Next, two biopotential leads (EEG electrodes) were individually secured to two miniature, stainless-steel screws threaded into the skull (relative to bregma: $\mathrm{AP}+1.0 \mathrm{~mm}, \mathrm{ML}+1.0 \mathrm{~mm}$, and $\mathrm{AP}-3.0 \mathrm{~mm}$, ML $-3.0 \mathrm{~mm}$ ) until making contact with dura. The screw-lead assemblies were secured to the skull with dental cement. The incision was closed with silk sutures, and lidocaine (2\%) and triple antibiotic ointment were applied. Mice received a postsurgical injection of an analgesic (buprenorphine $0.1 \mathrm{mg} / \mathrm{kg}$, IP) and recovered singly housed in standard Plexiglas home 
cages for 10 days before the transmitter battery was remotely activated.

\section{Physiological Recording (EEG, EMG, Locomotor Activity, Subcutaneous Temperature)}

Each home cage rested upon a RPC-1 PhysioTel receiver (DSI), which detected an AM radio signal emitted by the subcutaneous transmitter. The receivers were connected to a data exchange matrix (DSI) that constantly uploaded all recording data $(250 \mathrm{~Hz}$ sampling of EEG and EMG, and 10-s increments of mean subcutaneous temperature and total activity) to a computer with Dataquest ART Platinum analysis software (DSI). Zeitgeber time 0 (ZT0) was defined as 0700 (lights-on). Locomotor activity and temperature readings were analyzed with Dataquest ART Analysis software, and sleep, epileptiform activity, and EEG spectral power were analyzed using Neuroscore (DSI) with modules for sleep and spike-wave scoring. Temperature was analyzed as a change from daily mean for each mouse, because the subcutaneous placement of the transmitter can make these values susceptible to external factors (eg, air temperature).

\section{Sleep and Epileptiform Activity Analysis}

Sleep was manually scored in 10 -s epochs by three separate experimenters blinded to experiment conditions using spectral information from EEG, EMG amplitude, and activity measurements in a standardized manner (Franken et al, 1999). Wakefulness was characterized by elevated EMG amplitude, the presence of locomotor activity, and lowvoltage EEG amplitudes. SWS was characterized by the presence of high-amplitude delta $(0.5-4 \mathrm{~Hz})$ waves, low amplitude EMG, and absence of locomotor activity. PS was characterized by the presence of theta $(4-8 \mathrm{~Hz})$ waves, with EMG at or below baseline levels (indicative of muscle atonia). Automated sleep scoring from Neuroscore was run and each epoch was subsequently re-scored by a blinded experimenter. A minimum of two consecutive epochs in a different vigilance states was required to qualify as a transition between states. Artifacts were identified by extremely high amplitude or aberrant (non-physiological magnitude) signals, often in conjunction low transmitter signal strength. Epochs containing artifacts and epileptiform activity were removed for analysis of EEG spectral power, but retained and scored as being identical to the prior epoch for sleep stage and bout analysis. Bouts were considered as $20 \mathrm{~s}$ (ie, two consecutive epochs) or greater of wake, SWS, or PS, and considered 'broken' once interrupted by a bout of any other state. For bout distribution analysis, bouts were sorted into bins of increasing duration (Mochizuki et al, 2004). Using the Neuroscore Spikes module, the total number of spikes (defined as 3-20 $\times$ background and $>100 \mu \mathrm{V})$ was calculated from a 24 -h artifact-free recording period. Spike-wave discharges (SWDs) were identified by the Spike-Trains module (defined as $\geqslant 4$ spikes and $>0.5 \mathrm{~s}$ ) and verified by an experimenter. The relative EEG spectral power was quantified from the raw EEG signal using Fast Fourier Transformation and a Hamming signal window. The frequency bands analyzed were defined as Delta $(0.5-4 \mathrm{~Hz})$, Theta $(4-8 \mathrm{~Hz})$, Alpha $(8-12 \mathrm{~Hz})$, Beta $(16-24 \mathrm{~Hz})$, and low Gamma $(30-50 \mathrm{~Hz})$, with $50 \mathrm{~Hz}$ being the upper detection limit for the transmitters.

\section{Statistical Analyses}

Data were compared at two time points: 7 weeks and 12 weeks of age. Activity and temperature were analyzed in daily 3-h increments averaged over two 7-day periods, whereas sleep, epileptiform activity, and spectral power analyses were performed on two 24 -h periods. Comparisons were made using two-way (prenatal $\times$ postnatal treatment) or three-way (prenatal $\times$ postnatal treatment $\times$ time or powerband) analyses of variance with repeated measures when appropriate (eg, Figure 1b). Planned comparisons with Dunnett's multiple comparison correction were used to compare all groups with VV. Vigilance states, locomotor activity, and subcutaneous temperature were analyzed separately in the light and dark phase. Statistical analyses were performed with GraphPad Prism 7.0, SPSS 24.0, or SigmaPlot 13.0.

\section{RESULTS}

\section{Early-Life Immune Activation Has Lasting Effects on Locomotor Activity and Subcutaneous Temperature Patterns}

Prenatal and postnatal immune activation altered daily locomotor activity and temperature patterns. Activity and
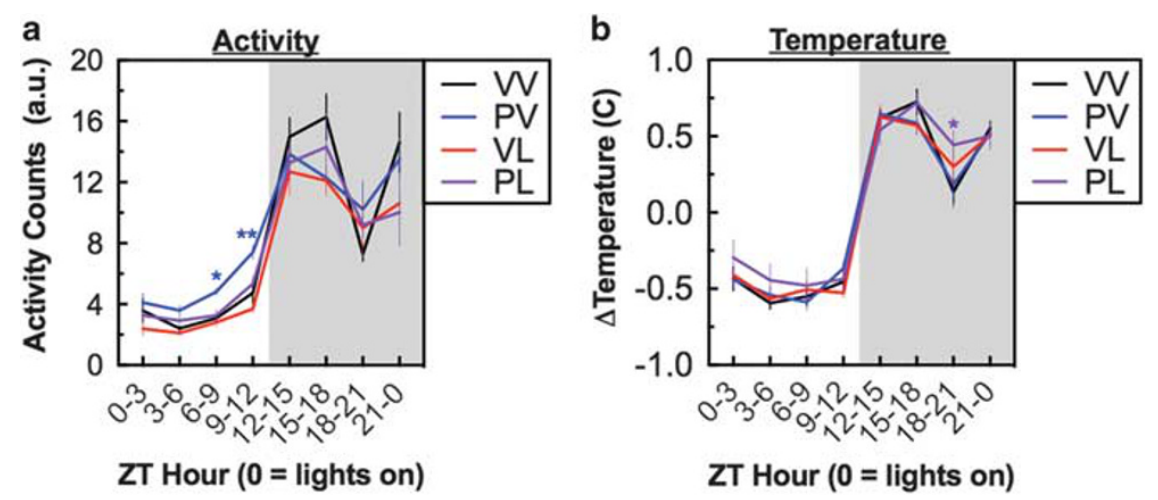

Figure 2 Treatment effects on circadian rhythms at week 7-8; data consolidated into 3-h time bins and averaged over a 7-day period (means \pm SEM). (a) Activity counts were lower during the dark phase in mice in the PV, VL, and PL treatment groups. (b) Relative temperature was higher during the dark phase in the PL treatment group. ${ }^{*} P<0.05$, $* * P<0.01$, compared with $\mathrm{VV}$, Dunnett's post hoc tests. 
temperature data were continuously collected over a 7-day period, collapsed, and analyzed as mean daily 3 -h periods. At the 7-weeks of age time point, during the light phase prenatal immune activation significantly increased motor activity ( $F$ $(1,20)=19.56, P<0.001$, for PV at ZT6-9: $P<0.05$, ZT9-12: $P<0.01$; Dunnett's tests), whereas postnatal immune activation was associated with decreased activity in the dark phase $(\mathrm{F}(1,20)=12.51, \quad P=0.002)$. (Figure 2a). However, this pattern did not persist at the 12-weeks of age time point, (prenatal: $\quad \mathrm{F}(1,20)=0.02, \quad P=.90 ; \quad$ and postnatal: $\mathrm{F}$ $(1,20)=2.63, P=0.12) \quad$ (Supplementary Figure S1A). In contrast, changes in subcutaneous temperature (which was normalized within each subject to account for differences in subcutaneous placement) were more persistent. At the 7week time point, temperature was higher in the PL ('twohit') mice during a distinct period in the dark phase (at ZT18-21, $P<0.05$, planned comparison) (Figure 2b), with a similar pattern of elevation at the 12-week time point (at ZT18-21, $P<0.01$, planned comparison) (Supplementary Fig S.1B).

\section{Early-Life Immune Activation Selectively Enhances Slow-Wave Sleep (SWS)}

Sleep was analyzed during a 24 -h period and was classified as active/wake, SWS, or paradoxical sleep (PS) in 10-s epochs. Perinatal immune activation affected sleep patterns at the 7-week time point, with postnatal LPS treatment being sufficient to produce effects, which were most evident during the light phase. During the light phase, there was a significant main effect of LPS on sleep time, with LPS exposure causing general increases in sleep time as indicated by a decrease in time awake (main effect of LPS: F $(1,20)=8.32, \quad P=0.0092)$ and PL-treated mice spending decreased time awake compared with controls (VV) (PL vs VV, $P<0.01$ ) (Figure 3b). The increases in sleep were owing to a selective increase in the proportion of time spent in SWS (main effect of LPS: $F(1,20)=9.663, P=0.0055$; and PL $v s \mathrm{VV}, P<0.05)$ (Figure 3e); there were no significant effects on time spent in PS (Figures $3 \mathrm{~h}$ and i). Patterns of effects during the dark phase were qualitatively similar, but none of the effects reached statistical significance (Figures $3 c, f$ and i). Sleep effects persisted through the 12-week time point: there was also a main effect of LPS $(\mathrm{F}(1,20)=8.06, P=0.01)$, with LPS exposure continuing to cause general decrease in time awake, although at this time point it was VL-treated mice that spent less time in awake than controls $(P<0.05)$ (Supplementary Figure S2A). As was the case with the earlier time point, there were the increases in sleep were owing to an increase in SWS (main effect of LPS: F $(1,20)=11.47, P=0.0029$; and both VL and PL $v s$ controls, $P$ 's $<0.05$ ) (Supplementary Figure S2C). Taken together, these data suggest that postnatal LPS is sufficient to produce longlasting increases in time spent in SWS, and that exposure to prenatal Poly I:C can enhance this effect.
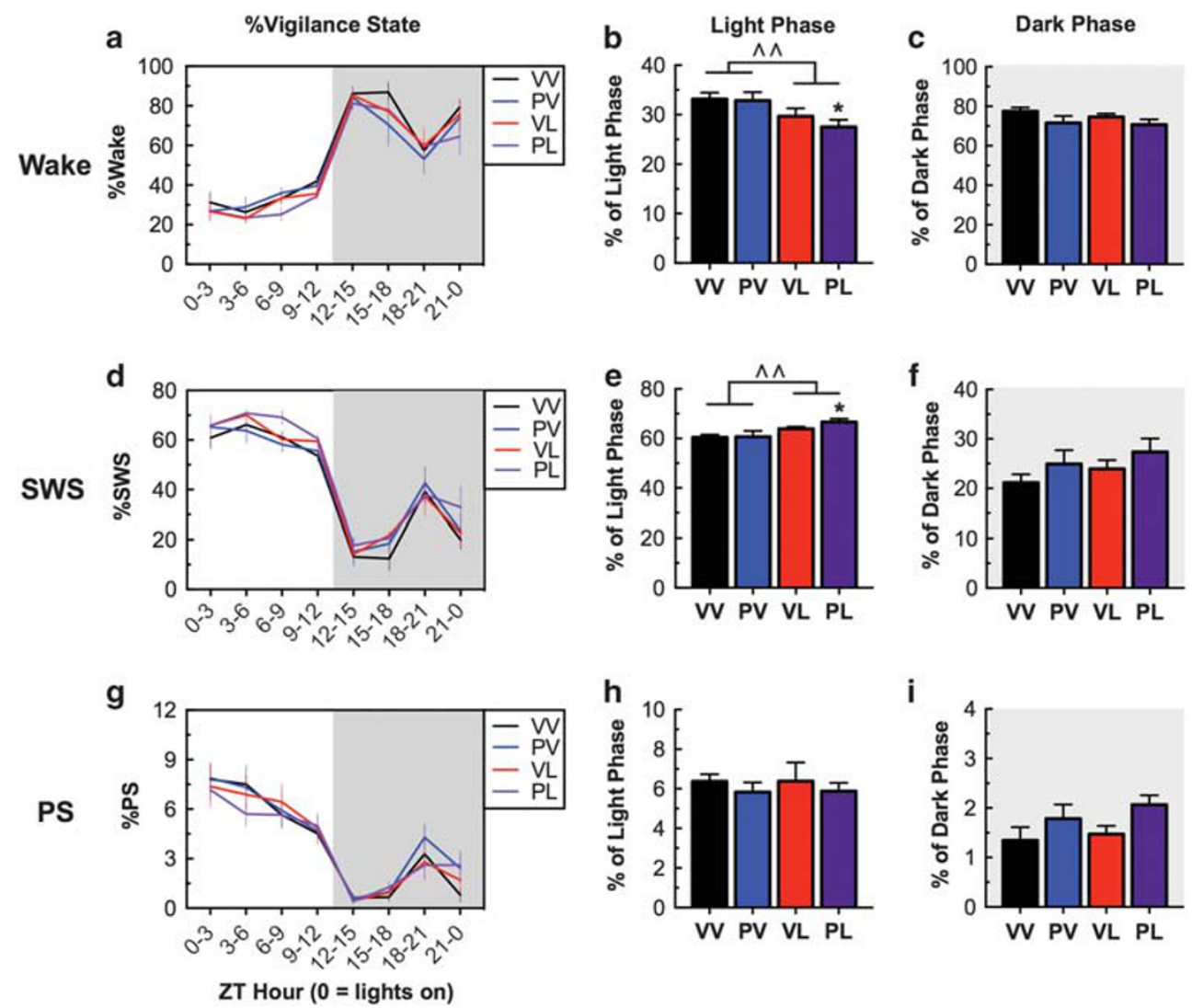

Figure 3 Treatment effects on vigilance states at Week 7; data reflect a 24-h period on Day 5 (means \pm SEM). (a,d,g) 3-h increments of vigilance state by treatment. (b,e,h) During the light phase, when mice are more likely to be sleeping, LPS-treated groups spent less time awake with largest effects in the PL ('two-hit') treatment group. Sleep effects were due to effects on SWS, with no changes in PS. (c,f,i) During the dark phase, when mice are more likely to be active, there were no statistically significant treatment effects. ${ }^{\wedge} P<0.05, \wedge \wedge P<0.0$ I, main effect; $* P<0.05$ compared with $\mathrm{V}$, Dunnett's post hoc tests. 
Table I Treatment Effects on the Duration and Count (number of bouts) (means \pm SEM) for the Various Vigilance States

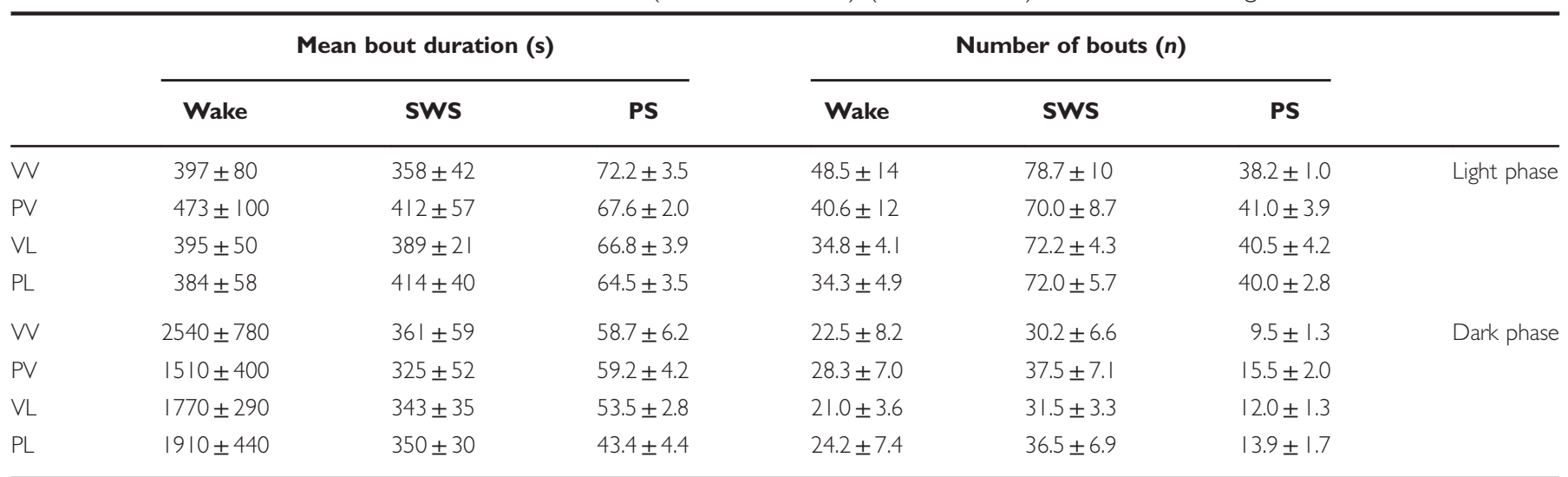

There were no significant differences, suggesting that these treatments did not produce sleep fragmentation.

To explore these changes in sleep architecture in more detail, we analyzed the average bout duration and the total number of bouts for each vigilance state. Increases in the number of bouts without corresponding increases in time are often considered to indicate sleep fragmentation (Wells et al, 2017). However, there were no statistically significant effects of perinatal immune activation on any of these measures (Table 1). A more detailed examination of the bout time distribution (ie, whether there differences in amount of time spent in shorter or longer bouts) indicated a small but statistically significant increase in shorter bouts of PS in both VL and PL mice (Bout Time $\times$ LPS, $F(4,100)=3.53, P=0.01$; and both VL and PL vs VV, $P^{\prime}$ s $<0.05$ ) (Supplementary Figure S3).

\section{Prenatal and Postnatal Immune Activation alter EEG Spectral Power}

Considering that alterations in resting-state EEG power are commonly found in ASD and other neuropsychiatric disorders, we also analyzed EEG spectral power. We focused on artifact- and epileptiform-free epochs from the 24-h period for each vigilance state, expressed as relative power normalized to VV mice. The largest effects were seen in PL mice, which showed dramatic shifts in spectral power compared with controls across vigilance states. During the active/wake state, treatment conditions significantly altered powerbands (powerband $\times$ prenatal exposure: $\mathrm{F}$ $(4,100)=9.13, P<0.001$; and powerband $\times$ postnatal exposure: $F(4,100)=3.97, P=0.005)$, with general increases in theta power (PV and VL vs VV, $P^{\prime} \mathrm{s}<0.05$, and $\mathrm{PL} v s \mathrm{VV}$, $P<0.01)$, and decreases in the PL group only ( $v s$ control) in Alpha $(P<0.05)$, Beta $(P<0.01)$, and low Gamma $(P<0.01)$ (Figure 4a). Likewise, treatment conditions also affected powerbands during SWS (powerband $\times$ prenatal exposure: $F$ $(4,100)=4.37, P=0.003)$, with decreases in Alpha power in PL mice $(P<0.05)$ (Figure $4 \mathrm{~b})$ and PS (powerband $\times$ prenatal exposure: $\mathrm{F}(4,100)=4.12, P=0.004$; and powerband $\times$ postnatal exposure, $\mathrm{F}(4,100)=2.51, P=0.047)$, with increases in Delta power $(P<0.01)$ and decreases in Alpha power $(P<0.01)$ in $\mathrm{PL}$ mice (Figure $4 \mathrm{c})$. Consolidating these findings into a simplified working model, 'two-hit' immune activation tended to increase lower frequencies (Delta, Theta) and decrease middle (Alpha) and higher (Beta, Gamma) frequencies (Figure 4d). There were qualitative similarities in these patterns at the 12-week time point in both active/wake (powerband $\times$ prenatal Exposure: $\mathrm{F}(4,100)=5.26, P<0.001$ ) and PS (powerband $\times$ prenatal exposure: $F(4,100)=6.04$, $P<0.001$ ), with $\mathrm{PL}$ mice having a reduction in Gamma power $(P<0.05)$ during active/wake and Alpha power $(P<0.01)$ during PS remaining statistically significant. In addition, several changes emerged at 12 -week time point during PS including an increase in Beta power $(P<0.01)$ in $\mathrm{PL}$ mice and a decrease in Alpha power $(P<0.05)$ in $\mathrm{PV}$ mice (Supplementary Figure S4A-C). These data suggest that combined treatment with prenatal Poly I:C and postnatal LPS produces effects that are greater than those seen with either treatment alone, and that several of the effects are persistent.

\section{Epileptiform Activity Following Postnatal LPS}

At the 7-week time point, we observed spontaneous highamplitude, $6-7 \mathrm{~Hz}$ poly-spikes on EEG recordings in LPStreated mice, prompting a more detailed analysis of epileptiform activity. These events had hallmark characteristics of SWDs: $6-7 \mathrm{~Hz}$, transient in nature, accompanied by arrest of behavior. These events occurred both during sleep (Example 1) and wakefulness (Example 2) (Figure 5a); SWDs occurring during wakefulness tended to be accompanied by cessation of EMG activity. Spectrograms of these events indicated peak frequency around $6-7 \mathrm{~Hz}$, and a minor harmonic at $\sim 13-$ $14 \mathrm{~Hz}$, consistent with previously reported spectral analysis of SWDs in mice (Ellens et al, 2009). Analysis revealed that SWDs were generated almost exclusively in mice that had received postnatal LPS treatment $(\mathrm{F}(1,20)=21.32, P<0.001$; and both VL and PL vs VV controls, $P$ 's $<0.01$ ) (Figure $5 \mathrm{~b}$ ). The increases in SWDs coincided with increases in the total number of high-amplitude spikes in LPS-treated mice ( $F$ $(1,20)=4.602, P=0.045$ ) (Figure $5 \mathrm{c}$ ), with a significant correlation between these end points $\left(R^{2}=0.494\right.$, $P=0.0001$; not shown). Interestingly, both SWDs and total spike number showed an apparent circadian rhythmicity, occurring more often during the light phase (when the mice are more likely to be asleep) than the dark phase (when they are more likely to be active) (main effects of time for SWDs: $\mathrm{F}(23,460)=3.46, \quad P<0.001 ;$ and Spikes: $\mathrm{F}(23,460)=3.20$, $P<0.001$ ) (Figures $5 \mathrm{~d}$ and e). The effects on SWDs persisted 

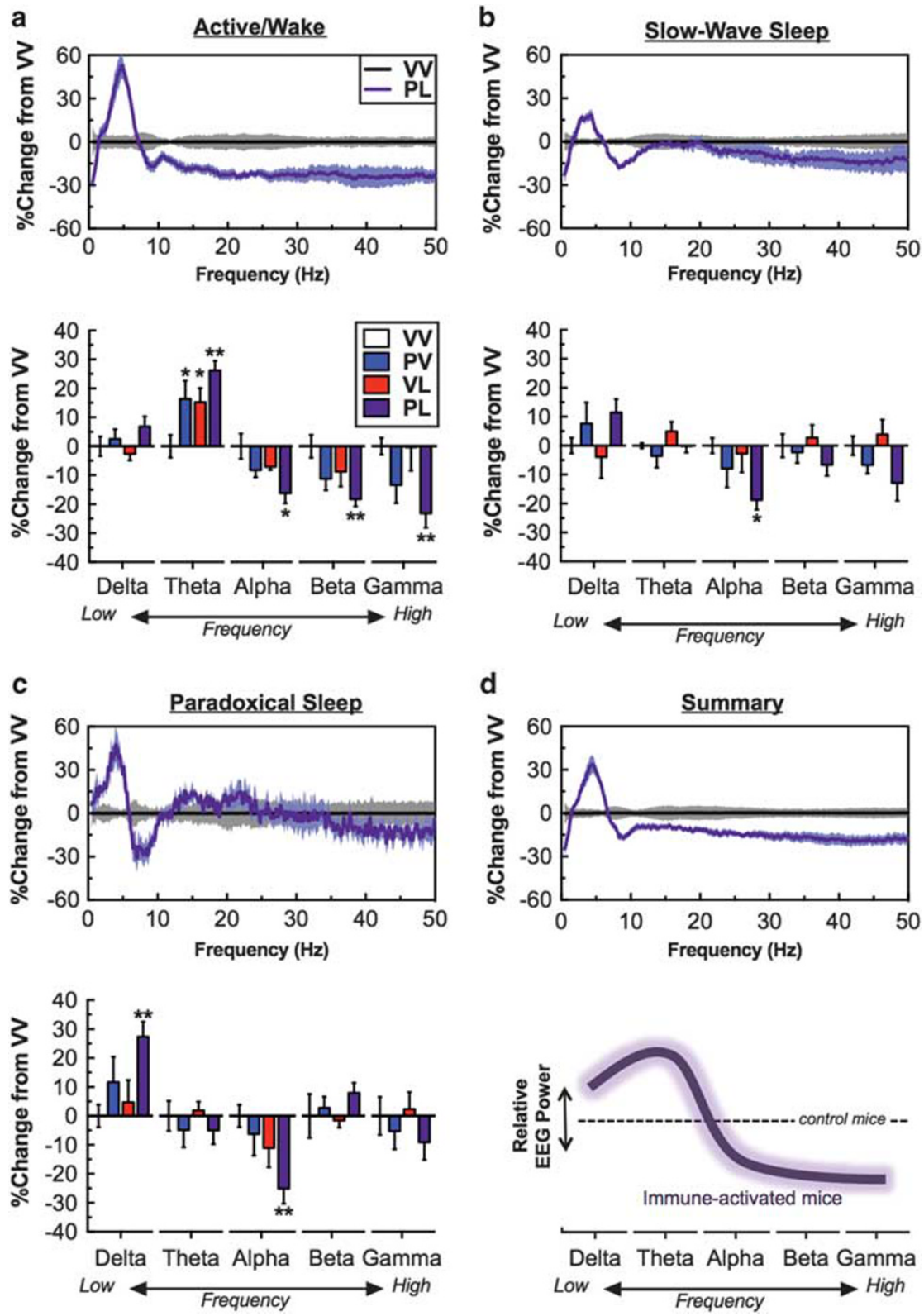

Figure 4 Treatment effects on EEG spectral power; data are consolidated over a 24-h period in I0-s epochs. For clarity, only PL-treatment mice are presented in the top panel, as effects were seen only in this group. Bottom panels show relative power bands of Delta $(0.5-4 \mathrm{~Hz})$, Theta $(4-8 \mathrm{~Hz})$, Alpha $(8-12 \mathrm{~Hz})$, Beta $(16-24 \mathrm{~Hz})$ and low Gamma $(30-50 \mathrm{~Hz})$, all normalized to $\mathrm{V}$-treated mice. (a, top/bottom) during active/wake, Theta power was higher but Alpha, Beta, and low Gamma power was lower in PL-treated mice. (b, top/bottom) During slow wave sleep (SWS), Alpha power was lower in PL-treated mice. (c) During paradoxical sleep, Delta was higher and Theta power was lower in PL-treated mice. (d) Simplified depiction of the general 'signature' of PL treatment on EEG spectral power. $* P<0.05$, ** $P<0.0$ I compared with $\mathrm{V}$, Dunnett's post hoc tests.

through the 12 -week time point $(\mathrm{F}(1,20)=7.557, P=0.012)$ (Supplementary Figure S5A), although the trend for an increase in the total number of spikes did not reach statistical significance (Supplementary Fig S5B). Together, these data suggest that postnatal LPS treatment is sufficient to produce long-lasting increases in spontaneous epileptiform activity that are not further enhanced by prenatal Poly I:C.

\section{DISCUSSION}

We show that early-life immune activation caused longlasting alterations in multiple physiological end points. These end points provide insight on abnormalities (eg, sleep disturbances, altered levels of general activity, seizures) that are often seen in ASD. The fact that the types of perinatal manipulations that produce the core behavioral signs of ASD (Custodio et al, 2017; Malkova et al, 2012) also produce common comorbid physiological disturbances increases confidence in the general approach as a model for ASD, and opens new avenues of research focusing on the pathophysiology and treatment of an immune subtype of this condition. Although more research is necessary to determine in detail how these abnormalities relate to the core features of ASD as measured in mice, our preliminary data (Missig et al, 2016) indicate similar trends in end points such as social interaction. 
a

\section{Example 1}

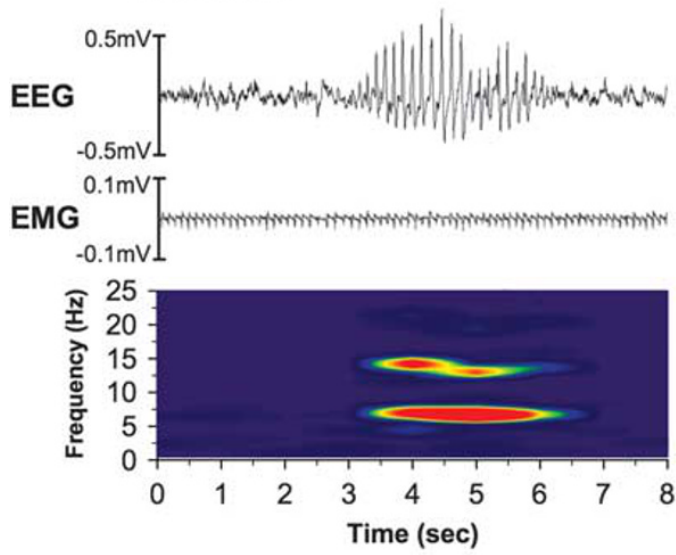

${ }^{b}$ Spike-Wave Discharges (SWDs)
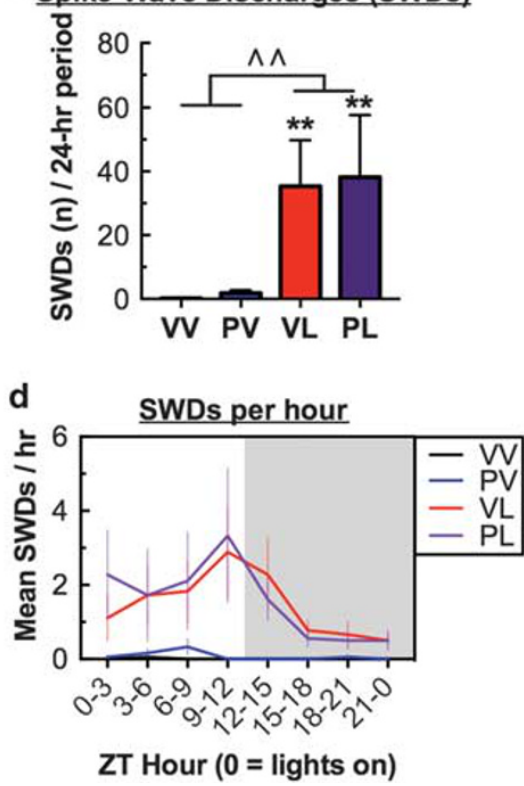

\section{Example 2}
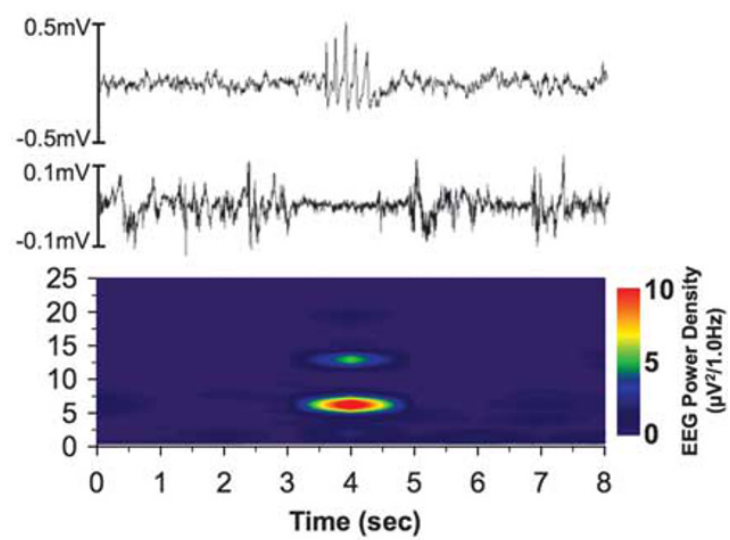

c
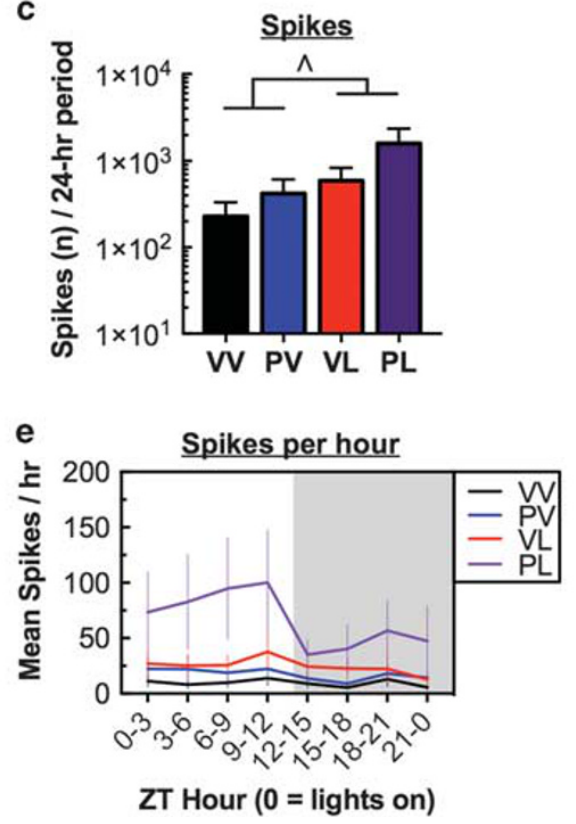

Figure 5 Treatment effects on EEG activity at Week 7; data reflect a 24-h period on Day 5 (Means \pm SEM). (a) Representative examples of spike-wave discharges (SWDs) observed during (left) sleep, with EEG activity occurring during a period of minimal muscle activity (EMG), and (right) wakefulness, with EEG activity corresponding to a brief period of arrested muscle activity. Spectrograms were created using a I-s Hanning window with $90 \%$ overlap. (b) LPS treatment was sufficient to produce large increases in SWDs, and (c) corresponding increases in the overall number of spikes. (d) Increases in SWDs were much more prevalent during the light phase, when mice are more likely to be sleeping, with (e) corresponding effects on the number of spikes. $\wedge P<0.05$, $\wedge \wedge P<0.01$, main effect; $* * * P<0.01$ with $W$, Dunnett's post hoc tests.

At the first time point we examined (7 weeks of age), prenatal Poly I:C alone had few effects on its own, although it increased activity during the light-phase. In contrast, postnatal LPS produced more robust effects, including the appearance of SWDs (which were extremely rare in control mice and in Poly I:C alone conditions), light-phase increases in SWS together with corresponding decreases in wakefulness, and dark-phase reductions in activity. The SWDs occurred throughout the day, but were most prevalent during the light phase, when mice are more likely to be sleeping. In general, the 'two-hit' (Poly I:C plus LPS) combined treatment produced the strongest effects on all of the metrics studied: in some cases the effects were only nominally different from those of LPS alone (eg, for SWS and SWDs), whereas in other cases statistically significant effects were seen only after combined treatment (eg, effects on spectral power). It is possible that we encountered 'ceiling effects' with some of these end points, such that seeing larger effects with the 'twohit' approach was simply not possible under our conditions. It is also possible that using a lower dose of LPS would have produced more modest effects on its own, enabling detection of more pronounced effects of the combined treatment. In preparing for these studies, we performed thorough doseeffect studies to identify an LPS dose that was high enough to produce an immune response in the 9-day old pups, while also being low enough to avoid causing sepsis and lethality. Despite these efforts, two pups died in the days following LPS administration ( 2 of $37,5.5 \%$ ) in the present studies. The dose-effect function was steep, and the dose tested here produced the most reliable effects across various lots of LPS, which can differ in potency. In addition, there was a nominal decrease in the average litter size following Poly I:C that did 
not reach statistical significance (Vehicle: 6.8 pups/litter $v s$ Poly IC: 5.3 pups/litter, $\mathrm{t}(10)=1.37, P=0.2)$. Regardless, our findings provide evidence that the 'two-hit' approach produces stronger changes in these end points than either of the individual treatments alone.

Many-though not all-of the effects of perinatal immune activation on these end points were long-lasting. Upon reanalysis (at 12 weeks of age), several of these effects remained evident, including the increases in SWS and appearance of SWDs in LPS-treated mice, and 'two-hit' effects on subcutaneous temperature and Gamma power. Several of these changes evolved over time: while the main effect of LPS on SWS and wakefulness persisted, the largest changes were seen in the LPS alone rather than the 'two-hit' group. Similarly, while the main effect of LPS on SWDs persisted, differences between LPS alone and the 'two-hit' treatment diminished. Only the effects of the 'two-hit' treatment on Gamma power during wakefulness and Alpha power during PS persisted, with new significant increases in Beta power during PS emerging over time. In contrast, the pattern of locomotor activity among treatment groups was not consistent between weeks 7 and 12. The reasons for these alterations in patterns are not clear, but obvious possibilities include a gradual reduction of any lingering effects of the acute inflammatory effects of the treatments themselves, the development of homeostatic counteradaptations in response to the induction of persistent changes in brain function, or more general effects of aging. Despite the changes in patterns over time, alterations in EEG power bands may reflect early-life inflammation induced changes in cortical synchrony.

Sleep dysregulation occurs frequently in ASD. The most commonly reported sleep problems are related to insomnia, including difficulties with sleep onset, maintenance, and duration (Richdale and Schreck, 2009). In two genetic rodent models of ASD (16p11.2 or CNTNAP2 deletion), mutant mice show increases in time awake together with either a consolidation or fragmentation of wakefulness (Angelakos et al, 2017; Thomas et al, 2017). These previous findings suggest that the increases in sleep that we observed following early-life immune activation reflect unique consequences of heightened neuroinflammatory processes. Indeed, it has been proposed that increased sleep is a stereotypic response to systemic inflammation, considering evidence that numerous cytokines promote sleep (Krueger et al, 2011). As examples, central or systemic administration of tumor necrosis factor alpha or Interleukin-1 beta increase SWS in a variety of species including mice, rats, primates, and humans (Krueger, 2008). Viewed in aggregate, many of the changes in the 'twohit' model resemble sickness behavior (Bilbo and Schwarz, 2012), including decreased activity, increased SWS, and altered temperature patterns. The presence of these signs may help to differentiate different ASD subtypes.

Although there is considerable evidence for comorbidities of ASD with seizures in humans, corresponding evidence from laboratory animals is currently limited. Seizure disorders are more prevalent in ASD compared with the general population, with estimates of comorbidities as high as $25-33 \%$ (Canitano, 2007). Although this is a broad association-with no one particular epilepsy syndrome or type being exclusively associated with ASD-focal seizures are often reported (El Achkar and Spence, 2015).
Importantly, epileptiform activity may be a key feature of ASD even in the absence of observable clinical seizures: in an EEG study of 889 ASD patients with no diagnosis of clinical epilepsy, $60 \%$ had abnormal epileptiform activity during sleep (Chez et al, 2006). It has been proposed that the cooccurrence of ASD and seizure disorders might result from common pathophysiologies, such as an alterations in excitatory/inhibitory balance (Buckley and Holmes, 2016). Indeed, lower seizure thresholds and/or higher numbers of spontaneous seizures are observed in several genetic mouse models of ASD (Stafstrom and Benke, 2015; Thomas et al, 2017). The epileptiform activity seen in the present studies resembles SWDs seen in EEG recordings from rodent models of absence seizure disorder, and the lack of EMG activity is similar to the behavioral arrest often observed during SWDs (Pearce et al, 2014). We also observed an increase in high-amplitude spikes in the LPS-treated mice. We acknowledge that this spike measure is automated and represents an inherently (but uncontrollably) noisy end point that may also include non-epileptiform activity, as evident by its occasional presence in the control mice. It is conceivable that the increase in spikes in PL mice could reflect, at least in part, increases in interictal spike activity or changes in the intrinsic properties of the EEG signals from these mice. Consistent with the interpretation that these changes are meaningful, however increases in spike was correlated with higher levels of SWDs. In addition, seizures have been previously associated with inflammatory mechanisms: early postnatal LPS administration in rats lowers thresholds for chemically induced seizures (Galic et al, 2008). Our studies provide new evidence that early-life immune system activation can result in spontaneous epileptiform activity, supporting a link between epilepsy and immune dysregulation.

Similar to the shifts in EEG spectral power observed in the current study, abnormal resting-state EEG power has been reported in EEG studies of humans with ASD. Although results have been variable, it has been proposed that the alteration in power structure in ASD is U-shaped, with increased power in lower frequencies (Delta, Theta), decreased middle frequencies (Alpha), and increased in high frequencies (Beta, Gamma) (Wang et al, 2013). The PLtreated mice in the present study approximate half of this shift, at low and middle frequencies, with the changes at high frequencies being inconsistent with this previous model. However, there are discrepancies within the literature, with reports of either increased or decreased Gamma power in ASD (Rojas and Wilson, 2014). Whether the heterogeneity in these findings reflects subtypes of ASD with different etiologies requires additional research. The origin and function of EEG oscillations remains unclear, although it is likely that they represent synchronous postsynaptic potentials resulting from complex neural circuit dynamics (Cohen, 2017). Individual components of EEG oscillation could have particular functions; for example, Alpha oscillations are thought to act as a functional inhibition, dependent on interneuron GABAergic feedback mechanisms (Jensen and Mazaheri, 2010). As such, the decreases in Alpha bands in the present studies may reflect impairment or imbalance of inhibitory feedback. Altered EEG oscillations could have utility as a biomarker that reflects underlying pathological changes in neural circuits in ASD and other neuropsychiatric conditions. 
The present findings provide justification for further work. We focused the present studies on male mice, considering the higher prevalence of ASD in males (Werling and Geschwind, 2013), but studies in females may provide insight on whether there are sex differences in sensitivity to these treatments or the severity of the effects. We gave prenatal Poly I:C and postnatal LPS treatment because of evidence showing that TLR3 expression is high but steadily decreasing during prenatal development, whereas TLR4 expression is low but steadily increasing over the same period (Barak et al, 2014). Postnatal administration of LPS also models the high risk of exposure to bacterial infections that occurs during this period of development. PND9 in a mouse approximates neurodevelopmental milestones, including brain growth, gliogenesis, and increases in axonal and dendritic density that are present in humans at full-term birth (Semple et al, 2013). In humans, labor produces over a 10fold increase of microbial invasion of the amniotic cavity (MIAC) (Romero et al, 2006; Seong et al, 2008), and pre-term birth is highly associated with MIAC and is a risk factor for ASD (Kuzniewicz et al, 2014; Romero et al, 2006). Furthermore, neonates are at high risk for bacterial infections, with an estimated incidence risk of $7.6 \%$ for severe infections (Seale et al, 2014). More work is needed to determine if the same pattern of effects would be seen with other combinations of treatments (prenatal LPS followed by postnatal Poly I: C, or two consecutive hits with Poly I:C and/or LPS). Similarly, while we show here that two environmental (immune) manipulations can produce these effects, future work might also examine whether 'two-hit' approaches that involve gene $\times$ environment interactions can produce comparable outcomes. We have recently used a similar approach to examine sleep and spectral power characteristics during a chronic social defeat stress (CSDS) regimen (Wells et al, 2017). CSDS resulted in a flattening of circadian rhythms, increases in PS and SWS, and alterations in EEG power bands. Although there are some between-study differences in the experimental design and analyses, the effects of CSDS and perinatal immune activation appear distinguishable on numerous levels (eg, effects on PS), suggesting different pathological mechanisms and highlighting the ways in which these various end points are uncoupled and free to vary independently. Our CSDS study highlights the utility of this EEG system for 'real-time' studies, to examine these end points before, during, and after an experimental manipulation. In comparison, this current study demonstrates the suitability of this system for evaluating changes in baseline that occur over long periods of time.

A 'multi-hit' theory was originally proposed in the context of understanding the causes of cancer (Knudson, 1971) and may provide a useful heuristic for understanding how immune system activation might increase ASD risk in some cases but not others (McDougle et al, 2015). Here, we show that a carefully timed sequence of two environmental hits against a stable genetic background can produce persistent physiological disturbances that often accompany the core signs of ASD. The combined Poly I:C plus LPS treatment produced the largest effects on EEG spectral power. Collapsing the effects over all phases of the sleep-wake continuum yields a potential 'EEG signature' of immune-related ASD, with elevations in low power bands and reductions in high power bands. The fact that only some elements of this signature remained evident at the extended (12-week) time point raises the possibility that developmental- or age-related factors can play a role in these metrics. New screening methodologies that identify patients with probable immune-related ASD would make it possible to determine if similar EEG signatures exist in humans, differentiating it from other subtypes of ASD (Wang et al, 2013). This type of advance would facilitate the diagnosis of the condition and the development of more targeted treatments for the various subtypes of ASD, as well as conditions that share common signs (eg, seizure disorders).

\section{FUNDING AND DISCLOSURE}

This research was funded by awards from the Robert and Donna Landreth Family Fund, the Nancy Lurie Marks Family Foundation, and the Teamsters Local 25 Autism Fund. The authors have no disclosures relevant to this research. Statistical analyses were conducted with support from Harvard Catalyst/The Harvard Clinical and Translational Science Center (National Center for Research Resources and the National Center for Advancing Translational Sciences, National Institutes of Health Award UL1 TR001102) and financial contributions from Harvard University and its affiliated academic healthcare centers. The content of this report is solely the responsibility of the authors and does not necessarily represent the official views of Harvard Catalyst, Harvard University and its affiliated academic healthcare centers, or the National Institutes of Health. The authors declare no conflict of interest.

\section{ACKNOWLEDGMENTS}

We thank Vadim Bolshakov, Kwang-Soo Kim, Woori Kim, and Yan Li for insightful discussions on this line of research.

\section{REFERENCES}

Angelakos CC, Watson AJ, O'Brien WT, Krainock KS, Nickl-Jockschat T, Abel T (2017). Hyperactivity and malespecific sleep deficits in the $16 \mathrm{p} 11.2$ deletion mouse model of autism. Autism Res 10: 572-584.

Barak B, Feldman N, Okun E (2014). Toll-like receptors as developmental tools that regulate neurogenesis during development: an update. Front Neurosci 8: 272.

Bilbo SD, Schwarz JM (2012). The immune system and developmental programming of brain and behavior. Front Neuroendocrinol 33: 267-286.

Buckley AW, Holmes GL (2016). Epilepsy and autism. Cold Spring Harb Perspect Med 6: a022749.

Canitano R (2007). Epilepsy in autism spectrum disorders. Eur Child Adolesc Psychiatry 16: 61-66.

Chez MG, Chang M, Krasne V, Coughlan C, Kominsky M, Schwartz A (2006). Frequency of epileptiform EEG abnormalities in a sequential screening of autistic patients with no known clinical epilepsy from 1996 to 2005. Epilepsy Behav 8: 267-271.

Cohen MX (2017). Where does EEG come from and what does it mean? Trends Neurosci 40: 208-218.

Custodio CS, Mello BSF, Filho A, de Carvalho Lima CN, Cordeiro RC, Miyajima F et al (2017). Neonatal immune challenge with lipopolysaccharide triggers long-lasting sex- and age-related behavioral and immune/neurotrophic alterations in mice: 
relevance to autism spectrum disorders. Mol Neurobiol (e-pub ahead of print).

El Achkar CM, Spence SJ (2015). Clinical characteristics of children and young adults with co-occurring autism spectrum disorder and epilepsy. Epilepsy Behav 47: 183-190.

Ellens DJ, Hong E, Giblin K, Singleton MJ, Bashyal C, Englot DJ et al (2009). Development of spike-wave seizures in $\mathrm{C} 3 \mathrm{H} /$ HeJ mice. Epilepsy Res 85: 53-59.

Estes ML, McAllister AK (2016). Maternal immune activation: implications for neuropsychiatric disorders. Science 353: 772-777.

Franken P, Malafosse A, Tafti M (1999). Genetic determinants of sleep regulation in inbred mice. Sleep 22: 155-169.

Galic MA, Riazi K, Heida JG, Mouihate A, Fournier NM, Spencer SJ et al (2008). Postnatal inflammation increases seizure susceptibility in adult rats. J Neurosci 28: 6904-6913.

Gupta S, Ellis SE, Ashar FN, Moes A, Bader JS, Zhan J et al (2014). Transcriptome analysis reveals dysregulation of innate immune response genes and neuronal activity-dependent genes in autism. Nat Commun 5: 5748.

Hornig M, Bresnahan MA, Che X, Schultz AF, Ukaigwe JE, Eddy ML et al (2017). Prenatal fever and autism risk. Mol Psychiatry (epub ahead of print).

Jensen O, Mazaheri A (2010). Shaping functional architecture by oscillatory alpha activity: gating by inhibition. Front Hum Neurosci 4: 186.

Jeste SS (2011). The neurology of autism spectrum disorders. Curr Opin Neurol 24: 132-139.

Jiang HY, Xu LL, Shao L, Xia RM, Yu ZH, Ling ZX et al (2016). Maternal infection during pregnancy and risk of autism spectrum disorders: a systematic review and meta-analysis. Brain Behav Immun 58: 165-172.

Kawai T, Akira S (2010). The role of pattern-recognition receptors in innate immunity: update on Toll-like receptors. Nat Immunol 11: $373-384$

Knudson AG Jr. (1971). Mutation and cancer: statistical study of retinoblastoma. Proc Natl Acad Sci USA 68: 820-823.

Krueger JM (2008). The role of cytokines in sleep regulation. Curr Pharm Des 14: 3408-3416.

Krueger JM, Majde JA, Rector DM (2011). Cytokines in immune function and sleep regulation. Handb Clin Neurol 98: 229-240.

Kuzniewicz MW, Wi S, Qian Y, Walsh EM, Armstrong MA, Croen LA (2014). Prevalence and neonatal factors associated with autism spectrum disorders in preterm infants. J Pediatr 164: 20-25.

Lezak KR, Missig GM, Carlezon WAJ (2017). Behavioral methods to study anxiety in rodents. Dialogues Clin Neurosci 19: 181-191.

Machado CJ, Whitaker AM, Smith SE, Patterson PH, Bauman MD (2015). Maternal immune activation in nonhuman primates alters social attention in juvenile offspring. Biol Psychiatry 77: 823-832.

Malkova NV, Yu CZ, Hsiao EY, Moore MJ, Patterson PH (2012). Maternal immune activation yields offspring displaying mouse versions of the three core symptoms of autism. Brain Behav Immun 26: 607-616.

Masi A, Quintana DS, Glozier N, Lloyd AR, Hickie IB, Guastella AJ (2015). Cytokine aberrations in autism spectrum disorder: a systematic review and meta-analysis. Mol Psychiatry 20: 440-446.

McDougle CJ, Landino SM, Vahabzadeh A, O'Rourke J, Zurcher NR, Finger BC et al (2015). Toward an immune-mediated subtype of autism spectrum disorder. Brain Res 1617: 72-92.

Miller AH, Haroon E, Felger JC (2017). the immunology of behavior-exploring the role of the immune system in brain health and illness. Neuropsychopharmacology 42: 1-4.
Missig GM, Alexander AJ, Mokler EL, Robbins JO, Sheets ME, Finger BC et al (2016). Altered behavior, sleep and epileptiform activity in a perinatal immune acivation mouse model of autism spectrum disorder (ASD). Neuropsychopharmacology 41: S474-S475.

Mochizuki T, Crocker A, McCormack S, Yanagisawa M, Sakurai T, Scammell TE (2004). Behavioral state instability in orexin knockout mice. J Neurosci 24: 6291-6300.

Pearce PS, Friedman D, Lafrancois JJ, Iyengar SS, Fenton AA, Maclusky NJ et al (2014). Spike-wave discharges in adult SpragueDawley rats and their implications for animal models of temporal lobe epilepsy. Epilepsy Behav 32: 121-131.

Richdale AL, Schreck KA (2009). Sleep problems in autism spectrum disorders: prevalence, nature, \& possible biopsychosocial aetiologies. Sleep Med Rev 13: 403-411.

Rojas DC, Wilson LB (2014). gamma-band abnormalities as markers of autism spectrum disorders. Biomark Med 8: 353-368.

Romero R, Espinoza J, Goncalves LF, Kusanovic JP, Friel LA, Nien JK (2006). Inflammation in preterm and term labour and delivery. Semin Fetal Neonatal Med 11: 317-326.

Seale AC, Blencowe H, Manu AA, Nair H, Bahl R, Qazi SA et al (2014). Estimates of possible severe bacterial infection in neonates in sub-Saharan Africa, south Asia, and Latin America for 2012: a systematic review and meta-analysis. Lancet Infect Dis 14: 731-741.

Semple BD, Blomgren K, Gimlin K, Ferriero DM, Noble-Haeusslein LJ (2013). Brain development in rodents and humans: identifying benchmarks of maturation and vulnerability to injury across species. Prog Neurobiol 106-107: 1-16.

Seong HS, Lee SE, Kang JH, Romero R, Yoon BH (2008). The frequency of microbial invasion of the amniotic cavity and histologic chorioamnionitis in women at term with intact membranes in the presence or absence of labor. Am J Obstet Gynecol 199: 375 e371-375 e375.

Smith SE, Li J, Garbett K, Mirnics K, Patterson PH (2007). Maternal immune activation alters fetal brain development through interleukin-6. J Neurosci 27: 10695-10702.

Stafstrom CE, Benke TA (2015). Autism and epilepsy: exploring the relationship using experimental models. Epilepsy Curr 15: 206-210.

Thomas AM, Schwartz MD, Saxe MD, Kilduff TS (2017). Cntnap2 knockout rats and mice exhibit epileptiform activity and abnormal sleep-wake physiology. Sleep 40 (e-pub ahead of print).

Wang J, Barstein J, Ethridge LE, Mosconi MW, Takarae Y, Sweeney JA (2013). Resting state EEG abnormalities in autism spectrum disorders. J Neurodev Disord 5: 24.

Wells AM, Ridener E, Bourdonais CA, Kim W, Pantazopoulos H, Caroll FI et al (2017). Effects of chronic social defeat stress on sleep and circadian rhythms are mitigated by kappa-opioid receptor antagonism. J Neurosci 0885-0817.

Werling DM, Geschwind DH (2013). Sex differences in autism spectrum disorders. Curr Opin Neurol 26: 146-153.

Wu S, Ding Y, Wu F, Li R, Xie G, Hou J et al (2015). Family history of autoimmune diseases is associated with an increased risk of autism in children: a systematic review and meta-analysis. Neurosci Biobehav Rev 55: 322-332.

Yin P, Liu J, Li Z, Wang YY, Qiao NN, Huang SY et al (2013). Prenatal immune challenge in rats increases susceptibility to seizure-induced brain injury in adulthood. Brain Res 1519: 78-86.

Supplementary Information accompanies the paper on the Neuropsychopharmacology website (http://www.nature.com/npp) 